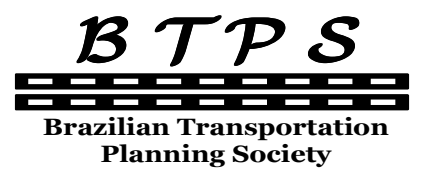

\author{
Journal of Transport Literature \\ Vol. 6, n. 1, pp. 139-156, Jan 2012 \\ Research Directory
}

JTL | RELIT

www.transport-literature.org ISSN 2238-1031

\title{
A programação matemática como ferramenta de auxilio na gestão das operações de uma empresa aérea
}

[Mathematical programming as a tool to assist in the management of an airline operations]

\author{
Marcelo Xavier Guterres*, Cláudio Sognálio Albano, Vera Lúcia Duarte Ferreira \\ Universidade do Estado do Rio de Janeiro (UERJ), Brazil, Universidade Federal do Pampa (UNIPAMPA), Brazil, \\ Universidade do Estado do Rio de Janeiro (UERJ), Brazil
}

Submitted 29 Aug 2011; received in revised form 16 Dec 2011; accepted 26 Jan 2012

\begin{abstract}
Resumo
As medidas de flexibilização impostas pelas autoridades aeronáuticas brasileiras, desde 1992, e intensificadas a partir de dezembro de 1997, através da concessão de maiores graus de liberdade no estabelecimento de tarifas e frequências por parte das empresas operadoras do transporte aéreo do País, propiciaram uma competição entre as empresas existentes até então nunca vistas, forçando-as a um novo tipo de posicionamento no mercado. 0 mercado competitivo fez com as empresas mudassem de uma política puramente operacional para uma política de negócios, visando à sobrevivência neste novo ambiente competitivo. Essas atuais características da indústria do transporte aéreo indicam uma ampla possibilidade de aplicação das técnicas de Pesquisa Operacional (PO) na solução dos problemas enfrentados pelas empresas. Primeiramente, este artigo busca mostrar as características atuais do mercado de transporte aéreo e os fatos que levaram a esta estrutura. A seguir é apresentada uma revisão de literatura que mostra as técnicas de Pesquisa Operacional que são utilizadas no fleet planning e finalmente é feito um estudo de caso que mostra uma aplicação de fleet assignment para uma pequena rede de voos regionais.
\end{abstract}

Palavras-Chave: programação linear; transporte aéreo.

\begin{abstract}
The easing measures imposed by the Brazilian aviation authorities, since 1992, and intensified from December 1997 through the award of higher degrees of freedom in setting rates and frequencies by the air transport business operators in the country, led a competition among existing hitherto unseen, forcing them to a new type of market positioning. The competitive market has caused companies to change their policy to a purely operational business policy in order to survive in this new competitive environment. These characteristics of the current air transport industry indicate a wide possibility of application of the techniques of Operations Research (OR) in the solution of problems faced by companies. First, this article seeks to show the current characteristics of the air transport market and the events that led to this structure. The following is a review of the literature shows that the Operations Research techniques that are used in fleet planninge and finally done a case study that shows an application of fleet assignment for a small network of regional flights.
\end{abstract}

Key words: linear programming; air transportation.

*Email: m.guterres@gmail.com.

\section{Recommended Citation}

Guterres, M. X., Albano, C. S. and Ferreira, V. L. D. (2012) A programação matemática como ferramenta de auxilio na gestão das operações de uma empresa aérea. Journal of Transport Literature, vol. 6, n. 1, pp. 139-156.

- JTL/RELIT is a fully electronic, peer-reviewed, open access, international journal focused on emerging transport markets and published by BPTS - Brazilian Transport Planning Society. Website www.transport-literature.org. ISSN 2238-1031. 


\section{Introdução}

O transporte aéreo brasileiro foi marcado por mudanças estruturais nos anos 1990, sobretudo devido a alterações no seu marco regulatório. Barreiras à entrada de companhias aéreas foram eliminadas, propiciando maior competitividade entre as empresas existentes. O novo ambiente no mercado levou às empresas aéreas a buscarem novos posicionamentos no mercado e mudarem de uma política puramente operacional para uma política de negócios.

Esta nova conjuntura de mercado extremamente competitivo permitiu à entrada de novas empresas. Um exemplo é a GOL linhas áreas, surgida em janeiro de 2001, com o conceito de low-cost. Basicamente, a estratégia desses novos competidores tem sido a busca permanente da eficiência operacional e do planejamento financeiro, visto que ambos mostram-se responsáveis pela maioria dos custos de operação desse negócio de grandes receitas, mas de baixa rentabilidade.

Assim, as atuais características da indústria do transporte aéreo indicam uma ampla possibilidade de aplicação de técnicas de Pesquisa Operacional (PO) na solução dos problemas enfrentados pelas empresas. As aplicações típicas da PO em empresas aéreas estão associadas ao planejamento operacional, cujo objetivo é a geração do timetable (programação horária de voos), que é o conjunto aeronave, ligação, escalas, frequência e horários de operação. Os problemas envolvidos neste processo são:

- Planejamento da frota: definição da composição da frota em um período (longo) prédeterminado;

- Alocação de aeronaves: definição das rotas que serão servidas, por tipo de aeronave da frota;

- Rotação de aeronaves: problema da alocação com a inclusão da dimensão tempo;

- Scheduling: tabelas de horários.

A disseminação dessas técnicas no transporte aéreo levou à formação de organizações dedicadas ao tema, como o The Airline Group of the International Federation of Operational Research Societies (www.agifors.org). A AGIFORS é uma sociedade profissional dedicada à promoção e aplicação da Pesquisa Operacional dentro da indústria aérea. A associação é 
constituída por profissionais de Pesquisa Operacional das companhias aéreas civis e indústrias relacionadas.

Assim, o presente artigo tem como objetivo abordar aspectos conceituais e resolutivos do uso das técnicas de PO no planejamento de pequenas empresas aéreas. Para tanto, será feito uma revisão da literatura e por fim será proposto um modelo de maximização de receita para uma pequena rede de voos. O resultados mostram que as atuais características da indústria do transporte aéreo indicam uma ampla possibilidade de aplicação de técnicas de otimização na solução dos problemas de planejamento e gestão que as firmas enfrentam.

\section{Estrutura do Mercado de Transporte Aéreo}

Em 1978, foi decretada a desregulamentação e a liberalização da indústria do transporte aéreo nos Estados Unidos da América (EUA) através da lei conhecida como Airline Deregulation Act. De acordo Wells (1996), esta medida teve como principal objetivo "encorajar, desenvolver e melhorar o sistema de transporte aéreo, que tem nas forças de mercado o determinante da qualidade, variedade e preços dos serviços aéreos”. As consequências imediatas deste processo no mercado norte-americano foram:

- Surgimentos de novas empresas, novas rotas e preços mais baixos;

- Aumento da competição;

- Maior número de assentos disponíveis;

- Menor taxa de ocupação;

- Menor taxa de lucratividade por passageiro;

- Falência de algumas empresas;

- Processo de fusão entre as empresas;

- Concentração dos negócios; 
A competição entre as empresas existentes foi intensificada por três inovações pioneiras que representaram mudanças radicais na aviação regular:

- Estrutura de rotas utilizando aeroportos hub (sistema hub-and-spoke);

- Desenvolvimento de sofisticado sistema de reservas;

- Estabelecimento de programas de frequência;

O sistema hub-and-spoke oferece ao passageiro uma maior escolha de voos, racionalizando as saídas a partir de um centro $(h u b)$. Os voos que costumavam servir apenas um mercado (ponto) passaram a servir vários. Assim, o hub-and-spoke, em resumo, é benéfico ao consumidor, na medida em que oferece mais opções de serviço para um maior número de destinos. Além disso, o número de passageiros que era obrigado a mudar de companhia ao fazer escalas reduziu drasticamente, o que também constitui uma melhoria no serviço prestado, pois passageiros, de uma forma geral, preferem conexões realizadas por vôos de uma mesma companhia.

A partir dos anos 80 as operações tipo hub-and-spoke se disseminaram largamente em outros países, alterando sensivelmente a estrutura dos serviços aéreos no mundo. A figura 01 ilustra as diferenças entre uma operação convencional e do tipo hub-and-spoke, e elenca as vantagens e desvantagens de uma operação hub-and-spoke (Guterres, 2002).

Figura 1: Operação convencional e tipo hub-and-spoke
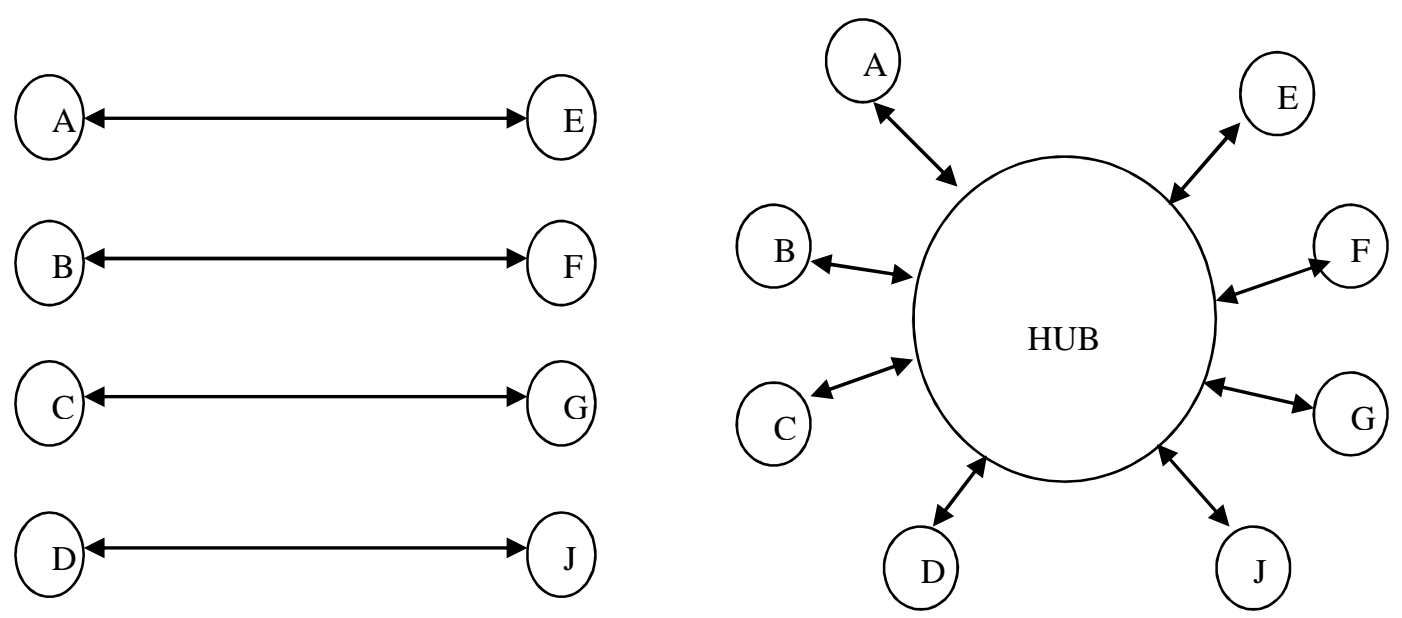

Fonte: Guterres (2002). 
As principais vantagens da utilização de um sistema hub-and-spoke são:

- Amplia o número potencial de serviços (pares de cidades);

- Maiores opções para os usuários;

- Possibilidade de maiores load-factors;

- Consolidação da empresa aérea no hub;

- Possibilidade de concentração de serviços;

As principais desvantagens são:

- Possibilidade de congestionamentos (chain reaction effect);

- Maior desequilíbrio no uso e demanda por facilidades;

- Maior desequilíbrio no uso e demanda por pessoal;

- Manuseio de bagagens;

Após praticamente vinte anos como forma preferencial da organização da oferta de vôos, as práticas tipo hub-and-spoke começam a enfrentar uma série de problemas, sendo o principal o congestionamento nos hubs (e suas consequências em termos de custo).

A expansão dos Computer Reservation Sytems (CRS) foi outro item que adquiriu grande importância devido à necessidade de controle mais rígido sobre as receitas, em função de margens de lucro mais estreitas, e também devido a grande participação das agências de viagens na venda das passagens.

Os principais benefícios da utilização dos CRS são:

- Melhor agilidade de operações com as agências de viagem;

- Estabelecimento de programas de gerenciamento de rentabilidade, na medida em que possuem banco de dados com as informações históricas dos passageiros (preferências, hábitos, renda, frequência);

- Estabelecimento de preços diferenciados e aumento da competição por preços;

A terceira consequência da abertura do mercado norte americano foi a criação dos programas de milhagem. Eles foram desenhados para aumentar a fidelidade do cliente à marca e diferenciar empresas internacionais, nacionais e regionais. Programas de fidelidade tornaram- 
se caros para administrar e benefícios começaram a impactar negativamente nas demonstrações financeiras. Os programas de fidelidade exercem papel perverso no desenho das estruturas de rotas, na medida em que rotas são mantidas ou adicionadas para evitar a perda de potenciais passageiros frequentes.

Estudos realizados por analistas americanos, como Bailey et al. (1986), Morrison \& Winston (1986), mostram que, em geral os resultados obtidos pela desregulamentação da indústria do transporte aéreo americano foram positivos. A desregulamentação aumentou a eficiência do sistema de prestação de serviços aéreos, reduziu os níveis tarifários do conjunto, aperfeiçoou o sistema de redes e o nível dos serviços no país. O sucesso desse processo e seu impacto mundial acabaram por influenciar decisivamente a exportação do modelo para outros mercados.

\section{A Utilização da Pesquisa Operacional em Empresas Aéreas}

As duas principais dificuldades de planejamento em uma companhia área dizem respeito ao planejamento da frota (fleet planning) e alocação das aeronaves (fleet assignment) na malha de vôos existentes.

O fleet planning é parte do processo de planejamento estratégico da empresa e é um dos mais difíceis de ser realizado. Ele visa responder quatro questões básicas: comprar uma aeronave, vender ou arrendar, aeronave nova ou usada, qual o tipo de aeronave e quando realizar a operação? As respostas a estas questões determinarão como o capital de investimento de longo prazo da companhia será utilizado. Os critérios que devem ser levados em conta para a composição da frota são: características de desempenho, impactos econômicos e financeiros, regulamentações ambientais, considerações de marketing e a realidade política.

A eficiência do processo de planejamento de frota experimentou um significativo aumento com a adoção, pela indústria aeronáutica, do conceito de família de aeronaves. Este conceito, utilizado por todos os principais fabricantes de aviões, implica em enormes ganhos em termos de flexibilidade e continuidade, em decorrência do desenvolvimento de uma série de aviões (família) a partir de uma configuração básica, aumentando-se (ou reduzindo) a capacidade 
pelo aumento (ou redução) da fuselagem, mantendo-se praticamente a mesma aviônica e controles aerodinâmicos.

Simpson (1968) propôs um modelo de programação linear de fleet planning com o objetivo de maximizar o lucro das companhias aéreas. O modelo de Simpson tem a seguinte função objetivo:

$$
\begin{aligned}
M A X Z_{T}=N C P & {\left[\sum Y_{r T} \cdot P_{r T}-\sum \sum D C_{r a T} \cdot n_{r a T}-I C_{T}\right] } \\
& +\sum \sum L P_{l a T} \cdot L_{l a T}-\sum I P_{i T} \cdot d_{I}-\sum \sum D E P_{p a T} \cdot A_{p a T} \\
& +\sum \sum\left(M v_{p a T}-B v_{p a t}\right) \cdot s_{p a T}
\end{aligned}
$$

Aonde:

$Z_{T}=$ lucro antes do imposto de renda no período $T$;

$N C P=$ número de ciclos por período;

$Y_{r T}=$ tarifa média/ciclo na rota $r$ no período $T$;

$P_{r T}=$ número médio de passageiros transportados por ciclo na rota $r$ no período $T$;

$D C_{r a T}=$ custo operacional direto médio/ciclo do avião tipo a na rota $r$ no período $T$ (menos depreciação);

$n_{r a T}=$ número médio de freqüências/ciclo do avião tipo a na rota $r$ no período $T$;

$I C_{T}=$ custo operacional indireto médio/ciclo do sistema no período $T$;

$L P_{l a T}=$ valor do pagamento de "leasing" efetuado ao início do período $T$ relativo a um avião do tipo a alugado no contrato l;

$L_{l a T}=$ número de aviões do tipo a alugados no contrato l existentes na frota no período $T$;

$d_{i}=i-$ ésima dívida pendente;

$I P_{i T}=$ proporção da dívida pendente $d_{i}$, cobrindo $T_{i}$ períodos, a ser paga como custo da dívida ao início do período T;

$D E P_{\text {Pat }}=$ custo de depreciação do avião tipo a comprado no plano de compras $p$ a incorrer no período $T$;

$A_{p a T}=$ número de aviões do tipo a comprados no plano de compra $p$ e existentes na frota no período T; 
Mv Pat $=$ previsão do valor de mercado para venda do avião do tipo a comprado no plano de compra p para o período T;

$B v_{p a T}=$ valor contábil do avião do tipo a comprado no plano de compra p para o período $T$;

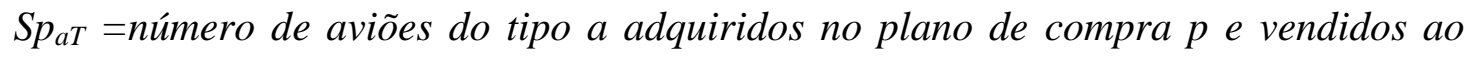
início do período T;

Cujas restrições são: load-factor máximo permitido, passageiros transportados como função da frequência, frequência total como soma de segmentos, frequências limitadas nos segmentos, disponibilidade de frota, frequência mínima por rota, limitações de decolagens por aeroporto. As limitações por período $T$ são: continuidade da frota, continuidade de caixa, endividamento máximo permitido, número de aviões comprados, número de aviões vendidos e número de aviões alugados.

Apesar da complexidade deste modelo, duas lacunas ainda podem ser identificadas: a não diferenciação do custo de introdução entre uma aeronave de tipo já existente na frota e outra completamente inédita, e, a ausência de uma taxa mínima de atratividade ou de retorno do capital investido. Ressalte-se que o volume de restrições neste modelo de planejamento de frota com o uso da programação linear praticamente obriga sua decomposição para a resolução.

Já os modelos matemáticos de fleet assignment são utilizados com o objetivo de distribuir a frota (produto dos modelos de fleet planning) em uma rede de linhas previamente conhecida. Ao contrário dos modelos de fleet planning, estes modelos são aplicáveis para horizontes mais próximos. Assim, os processos afetados por estas decisões de planejamento são: a programação da tripulação, a manutenção das aeronaves, o marketing e as operações. 
Além do conhecimento da composição da frota (número de aeronaves, por tipo), os modelos de fleet assignment requerem ainda dados e informações sobre:

- Demanda média para cada ligação, dentro de um período de tempo pré-fixado;

- Load-factor estimado para cada tipo de aeronave, em cada linha;

- Utilização média por tipo de aeronave;

- Parâmetros econômico-financeiros (tarifas, custos operacionais);

- Restrições de natureza operacional (capacidade dos aeroportos e comprimento de pista);

Apresenta-se, a seguir, um exemplo de modelo de Programação Linear, extraído de Simpson (1968). O objetivo do problema é alocar as aeronaves (a) às diversas rotas (R) minimizando o custo operacional direto global, para a operação diária da frota. Os parâmetros e variáveis deste modelo são:

$R=$ conjunto de rotas ligando origens $i$ a destinos $j$;

$a=$ identificador de cada tipo de aeronave da frota;

$P_{i j}=$ passageiros (média diária) transportados na rota ij;

$C D_{a i j}=$ custo operacional direto da aeronave tipo a, operando na rota ij;

$n_{a i j}=$ número diário de voos da aeronave tipo a na rota $i j$;

$L F_{a i j}=$ load-factor médio da aeronave tipo a, operando na rota ij;

$S a=$ capacidade (assentos) da aeronave tipo a;

$T B_{a i j}=$ tempo de bloco da aeronave tipo a, operando na rota $i j$;

$U_{a}=$ utilização média diária da aeronave tipo a;

$N_{a}=$ número de aeronaves do tipo a disponíveis na frota;

$N F_{i j}=$ número mínimo de frequências diárias oferecidas na rota ij;

$M D_{i}=$ número máximo de decolagens no aeroporto $i$; 
A função objetivo tem a seguinte forma:

$$
\min Z=\sum_{R} \sum_{a} C D_{a i j} \cdot n_{a i j}
$$

Já as restrições do modelo são:

$$
\begin{gathered}
\sum_{a} L F_{a i j} \cdot S_{a} \cdot n_{a i j}=P_{i j} \\
\sum_{R} T B_{a i j} \cdot n_{a i j} \leq U_{a} \cdot N_{a} \\
\sum_{j} n_{a i j}-\sum_{j} n_{a j i}=0 \\
\sum_{a} n_{a i j} \geq N F_{i j} \\
\sum_{a} \sum_{j} n_{a i j \leq M D_{i}}
\end{gathered}
$$

- A equação 03 representa o atendimento da demanda para cada rota ij;

- A equação 04 a disponibilidade de aeronaves para cada tipo (a);

- A equação 05 mostra a equação de continuidade para cada cidade $i$ e tipo (a), esta restrição estabelece que, em cada cidade atendida, o número de chegadas diárias de um determinado tipo de aeronave deve ser igual ao número de partidas;

- A equação 06 caracteriza a oferta mínima para cada rota ij;

- Por fim a equação 07 representa a capacidade dos aeroportos para cada aeroporto;

O modelo pode ser resolvido pelo método simplex, ou por um algoritmo específico para programação linear inteira. No primeiro caso (simplex), os valores fracionários que eventualmente sejam obtidos podem ser interpretados como valores médios durante o período de planejamento. Deve-se ressaltar que os conjuntos de restrições "oferta mínima" e "capacidade dos aeroportos" não precisam, necessariamente, serem impostos para cada rota e para cada aeroporto. 


\section{Modelagem e Projeção de Demanda numa Ligação}

Previsões de demanda desempenham um importante papel na operacionalização de diversos aspectos do gerenciamento da produção de uma empresa aérea. A estimativa do tráfego futuro é essencial no diz respeito ao planejamento da frota e alocação das aeronaves. Esses modelos são conhecidos como modelos de tráfego direcional e são voltados para mercados específicos (ex.: passageiros transportados na ligação São Paulo - Rio de Janeiro).

A primeira abordagem lógica para se projetar demanda de passageiros em uma rota é a de estudar a série temporal de dados e analisar a tendência de evolução. Esse procedimento, no entanto, é aplicável somente às rotas existentes e não leva em conta como diversos fatores econômicos, sociais e operacionais afetam a evolução do tráfego. É possível que, em rotas bem exploradas em regiões desenvolvidas social e economicamente, o uso de tendências históricas, para projeções num pequeno horizonte de tempo, seja prático e eficaz. Para o Brasil, no entanto, o exame das pequenas séries temporais disponíveis para o tráfego de origem-destino evidencia a inadequação desta técnica para quase a totalidade das ligações aéreas, pela própria dificuldade e risco de se estabelecer uma tendência.

Um modelo clássico para estimar o fluxo de passageiros entre duas cidades ou regiões é o conhecimento modelo gravitacional. Na sua forma original, apresentada no século XIX, o modelo incluía as seguintes variáveis para descrever o fluxo ferroviário de passageiros entre estações distintas, em que:

$N$ : número de passageiros;

P1 e P2: população das duas cidades;

D: distância entre as duas cidades;

$C$ : Constante a ser determinada;

O modelo matemático sendo expresso pela equação (8):

$$
\mathrm{N}=\frac{\mathrm{C}\left(\mathrm{P}_{1} \cdot \mathrm{P}_{2}\right)}{\mathrm{D}^{2}}
$$


Ainda que adequado para o objetivo ao qual se aplicou, o modelo original não é aplicável à previsão de tráfego aéreo, a médio e longo prazo. Motivo disto é que as variáveis independentes incluídas são exclusivamente magnitudes físicas e não refletem os fatores econômicos, técnicos ou sociais que influem na evolução do tráfego aéreo com o tempo. Modelos gravitacionais generalizados (ou modificados), do tipo:

$$
\mathrm{N}=\frac{\mathrm{C}\left(\mathrm{P}_{1} \mathrm{P}_{2}\right)^{\alpha}}{\mathrm{D}^{\beta}}
$$

Onde $C$, $\alpha$ e $\beta$ são coeficientes ajustados pelo método dos mínimos quadrados, são, até nossos dias, utilizados.

No Brasil, a análise do comportamento de fluxos de passageiros em ligações aéreas incorporou esta metodologia - modelos do tipo gravitacional generalizado, envolvendo variáveis relevantes das áreas de influência do par de cidades que definem a ligação. Tal metodologia, frequentemente utilizada nos estudos da ICAO, possui uma boa relação causal entre a variável dependente e as variáveis independentes, que refletem não só os fatores socioeconômicos como também os tecnológicos que possam explicar o desenvolvimento do tráfego aéreo e / ou ainda, os fatores característicos de oferta de transporte aéreo entre as duas localidades.

A demanda ou o tráfego entre um par qualquer de localidades toma a forma de um produto de variáveis socioeconômicas das áreas de influência das cidades servidas e de características tecnológicas e / ou de oferta de transporte aéreo entre as mesmas. A estrutura típica dos modelos gravitacionais generalizados utilizados no Brasil é a seguinte:

$$
\mathrm{T}_{\mathrm{ij}}=\alpha_{0} \cdot\left(\mathrm{M}_{\mathrm{i}} \cdot \mathrm{M}_{\mathrm{j}}\right)^{\beta_{1}} \cdot\left(\mathrm{C}_{\mathrm{ij}}\right)^{\beta_{2}}
$$


Onde:

$T_{i j}$ : volume de tráfego aéreo de passageiros entre as áreas de influência das localidades i e j;

$M_{i}$ e $M_{j}$ : variáveis socioeconômicas das áreas de influência das localidades i e j;

$C_{i j}$ : variável característica da oferta de transporte aéreo entre i e $j$;

$\alpha_{0}, \beta_{1}$ e $\beta_{2}$ : parâmetros a estimar.

O modelo exposto pode ser transformado e então ajustado por regressão linear, da seguinte forma:

$$
\ln \left(\mathrm{T}_{\mathrm{ij}}\right)=\alpha_{0}+\beta_{1} \ln \left(\mathrm{M}_{\mathrm{i}} \cdot \mathrm{M}_{\mathrm{j}}\right)+\beta_{2} \ln \left(\mathrm{C}_{\mathrm{ij}}\right)
$$

\section{Estudo de Caso}

Considerar-se-á uma empresa de transporte aéreo regional que atende as seguintes cidades: A, B, C e D que geram seis possíveis itinerários, ressaltados na tabela 01. Os trechos A-B e C-D é operado com uma aeronave de 160 lugares divididos em duas classes (executiva e econômica), já o trecho B-C é percorrido com uma aeronave de 140 lugares, que também apresenta duas opções de classes para os usuários do serviço (figura 02).

Figura 2: Itinerários da Empresa

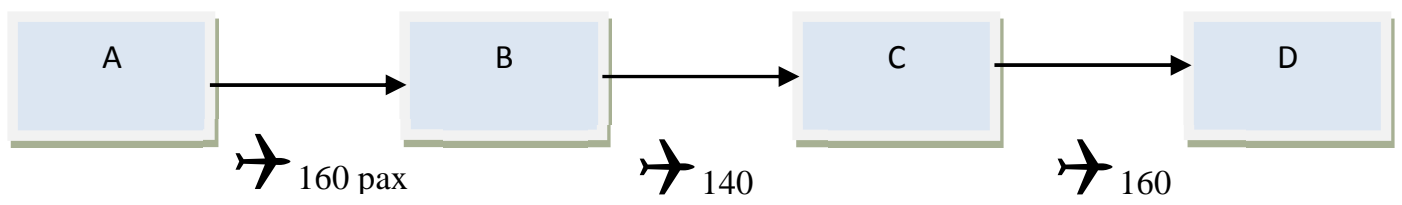

Considerando-se que as estimativas das demandas nas ligações foram obtidas com aplicação de um modelo de tráfego direcional, e as aeronaves alocadas são frutos do fleet planning, e que a firma não pratica overbooking, estratégia que implica dispor para venda um número de assentos maior do que o existente, para compensar os efeitos dos cancelamentos, formular-seá um modelo de fleet assignment para a maximização da receita desta rede. 
Tabela 1: Dados de Demanda e Tarifa

\begin{tabular}{|c|c|c|c|c|}
\hline Itinerários & $\begin{array}{l}\text { Tarifa (\$) } \\
\text { Classe Y }\end{array}$ & $\begin{array}{l}\text { Tarifa (\$) } \\
\text { Classe C }\end{array}$ & $\begin{array}{l}\text { Demanda } \\
\text { Classe Y }\end{array}$ & $\begin{array}{l}\text { Demanda } \\
\text { Classe C }\end{array}$ \\
\hline A-B & 300,00 & 400,00 & 90 & 30 \\
\hline $\mathrm{B}-\mathrm{C}$ & 200,00 & 250,00 & 40 & 50 \\
\hline C-D & 300,00 & 360,00 & 50 & 25 \\
\hline $\mathrm{A}-\mathrm{C}$ & 400,00 & 480,00 & 70 & 30 \\
\hline A-D & 700,00 & 840,00 & 80 & 40 \\
\hline B-D & 400,00 & 450,00 & 60 & 30 \\
\hline
\end{tabular}

O modelo de programação linear proposto apresenta a seguinte função objetivo:

$$
\max L=\sum_{i=1}^{m} \sum_{j=i+1}^{n} T y_{i j} . X y_{i j}+\sum_{i=1}^{m} \sum_{j=i+1}^{n} T c_{i j} X c_{i j}
$$

Aonde:

$$
\begin{aligned}
& \text { y- classe econômica } \\
& \text { c-classe executiva } \\
& T y_{i j}=\text { valor da tarifa da classe y da origem i para o destino } j \text {; } \\
& T c_{i j}=\text { valor da tarifa da classe } c \text { da origem i para o destino j; }
\end{aligned}
$$

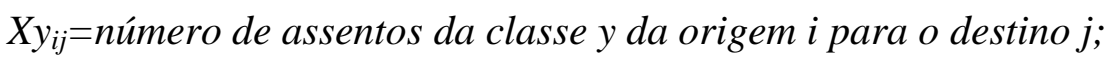

$$
\begin{aligned}
& X c_{i i}=\text { número de assentos da classe } c \text { da origem i para o destino j; } \\
& m=n u ́ m e r o \text { de origens; } \\
& n=\text { número de destinos }=m+1 \text {; } \\
& L=\text { lucro }
\end{aligned}
$$

Sujeito as seguintes restrições:

- Capacidade das aeronaves: o número total de passageiros nos trechos não pode exceder a capacidade da aeronave no trecho;

- Demanda: os assentos que serão alocados em cada classe por itinerário não pode exceder a demanda estimada para a classe; 
Para resolver este problema de programação linear foi utilizado o software LINDO (Linear, Interactive, and Discrete Optimizer). Este programa é uma ferramenta poderosa na solução de problemas de otimização. No anexo 01 pode ser visto a entrada de dados e a resposta obtida.

Tabela 2: Resultados do Modelo de Programação Linear proposto para o Estudo de caso

\begin{tabular}{|c|c|c|c|c|r|}
\hline Itinerários & $\begin{array}{c}\text { Tarifa }(\$) \\
\text { Classe Y }\end{array}$ & $\begin{array}{c}\text { Tarifa (\$) } \\
\text { Classe C }\end{array}$ & $\begin{array}{c}\text { Assentos } \\
\text { Classe Y }\end{array}$ & $\begin{array}{c}\text { Assentos } \\
\text { Classe C }\end{array}$ & Receita \\
\hline A-B & 300,00 & 400,00 & 75 & 30 & $34.500,00$ \\
\hline B-C & 200,00 & 250,00 & 5 & 50 & $13.500,00$ \\
\hline C-D & 300,00 & 360,00 & 50 & 25 & $24.000,00$ \\
\hline A-C & 400,00 & 480,00 & 0 & 0 & 0,00 \\
\hline A-D & 700,00 & 840,00 & 15 & 40 & $44.100,00$ \\
\hline B-D & 400,00 & 450,00 & 0 & 30 & $13.500,00$ \\
\hline & & & & Total & $129.600,00$ \\
\hline
\end{tabular}

Conclui-se que a solução ótima está em distribuir primeiramente toda a demanda média de passageiros na classe executiva. Como podemos ver na tabela 02 , apenas no itinerário A- C não foi alocado à demanda média da classe executiva, nos demais itinerários foram reservados o número de assentos equivalentes a demanda média da classe executiva, isto ocorre, devido ao fato que a classe executiva gera mais receita, pois possui uma maior tarifa.

No modelo proposto de programação linear para maximização da receita da rede de voos da empresa aérea regional, percebe-se que não existe uma restrição ativa quanto a um número determinado de assentos na classe executiva e econômica. Logo, no modelo usado, um aumento no número de assentos na classe executiva e econômica não implicaria nenhuma alteração na resposta, pois o modelo tem como restrição ativa a demanda média de passageiros na classe executiva.

Caso o modelo tivesse como restrição ativa o número de assentos das classes, uma eventual mudança no interior da aeronave ocasionaria mudança na maximização da receita, pois um aumento na classe executiva ocasionaria um aumento na receita, e caso contrario uma diminuição na receita. 


\section{Conclusão}

As características da indústria do transporte aéreo indicam uma ampla possibilidade de aplicação de técnicas de PO, pois:

- Sendo ela de capital intensiva em relação ao veículo, e dada a indivisibilidade da oferta, os investimentos em termos de frota de aeronaves são de grande monta. E mais: são investimentos de longa maturação (pela quase inexistência de "entrega imediata" das aeronaves). Assim, uma decisão errada pode comprometer irremediavelmente a situação econômico-financeira da empresa aérea;

- O usuário do modo aéreo prioriza o tempo. Desta forma, o planejamento e a coordenação da rede de processos e parcerias de modo a minimizar o tempo de produção são fundamentais;

- A dimensão relativa (pequena) do transporte aéreo facilita a modelagem matemática, sua validação e controle.

Desta forma, as empresas aéreas, que buscam maximizar a utilização de suas aeronaves (consequentemente, o menor tempo de solo possível) e dos equipamentos ou serviços de rampa, além de oferecer o melhor nível de conforto para seus usuários, obrigatoriamente devem-se valer das técnicas de Pesquisa Operacional para atingirem tal objetivo. 


\section{Referências}

Bailey, E. E., Grahan, D.R. e Kaplan, D.P. (1986) Deregulating The Airlines. Cambridge: The MIT Press.

Guterres, M. X. (2002) Efeitos da Flexibilização do Transporte Aéreo Brasileiro sobre a Concentração da Indústria, Dissertação (Mestrado em Ciências) - Instituto Tecnológico de Aeronáutica, São José dos Campos, SP.

Lopes, D.R. (1990) Contribuição à modelagem do problema do planejamento da operação de pátios de aeronaves em aeroportos. Tese de Doutorado, EPUSP, São Paulo.

Morrison, S. e Winston, C. (1996) The Evolution of the Airline Industry. Washington Brookins Institution.

Simpson, R. (1968) Scheduling and routing models for airline systems. MIT Flight Transportation Laboratory, Report FTL-R63-3, MIT, USA.

Wells, A.T. (1996) Airport Planning \& Management . McGraw-Hill, 3rd Edition, New York. 


\section{Anexo}

1-Tela inicial de entrada dos dados

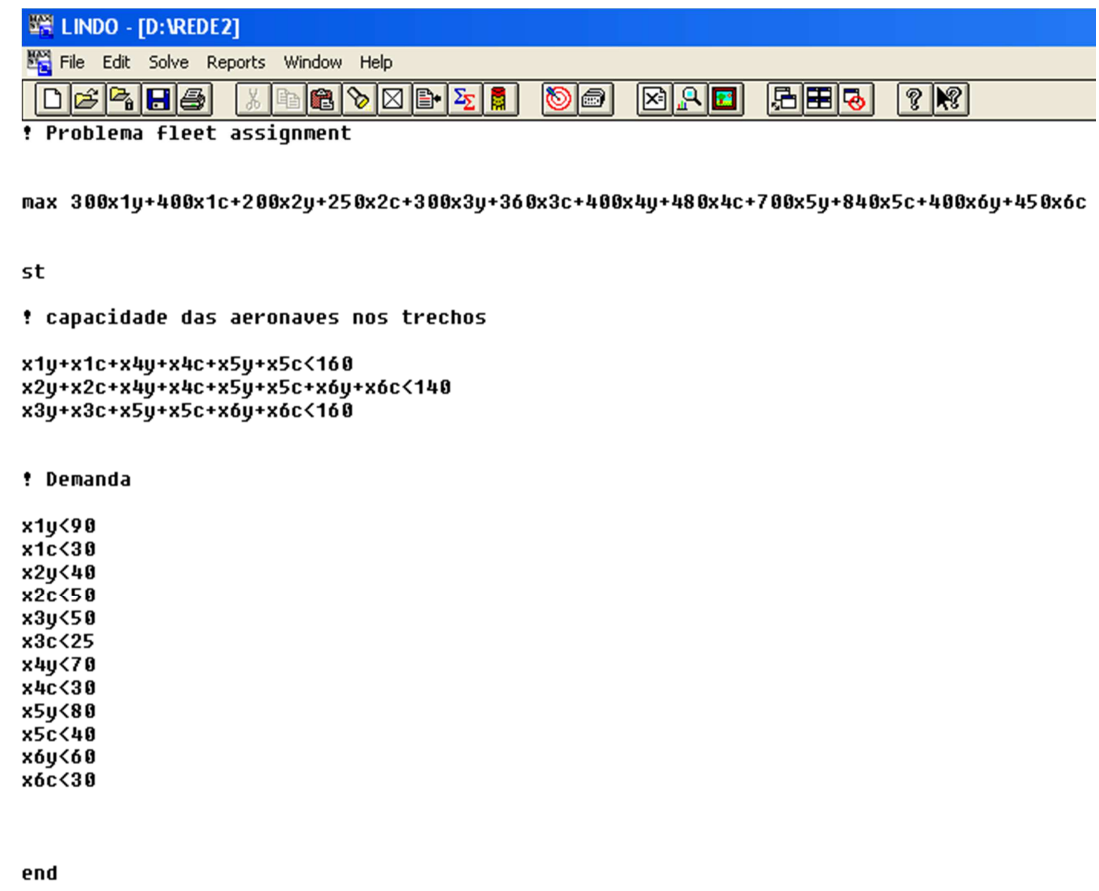

2- Solução do modelo

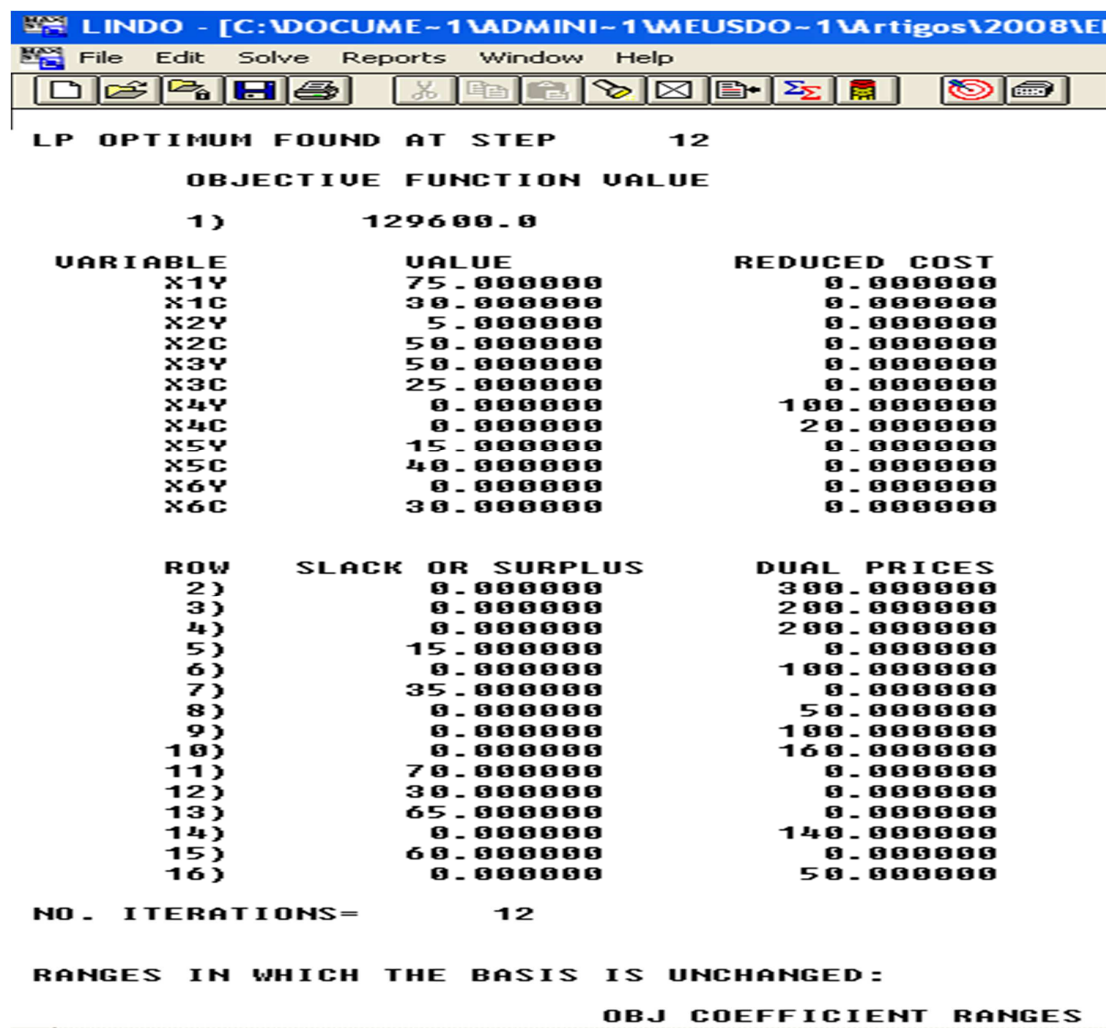

